\title{
Oxygen Utilization and Hemodynamic Response during Exercise in Ghildren after Fontan Procedure
}

\author{
Takeshi Mryarri, MD, Motohiro Kawauchi, MD, \\ Shinichi Takamoto, MD, Osamu Morizuki, ${ }^{1} \mathrm{MD}$, \\ and Akira Furuse, ${ }^{2} \mathrm{MD}$
}

\section{SuMMary}

Eight patients, 9.1 to 16.5 years of age, were studied 2.8 to 8.5 years after Fontan operation. Oxygen utilization was determined during upright bicycle exercise. The cardiac index and stroke index were measured by echocardiography and the anaerobic threshold was determined. The results were compared with 10 patients after surgical closure of the atrial septal defect. Anaerobic threshold (AT) in Fontan patients was lower than in the control subjects. Oxygen consumption at each stage of exercise was significantly lower in the Fontan group compared with the control subjects. From the beginning of exercise until AT, the increase in stroke index was lower in the Fontan patients than in the control subjects. After that point, the stroke index decreased signifcantly in the Fontan patients while it remained almost at the same level in the control subjects. Significant correlations were observed between the oxygen pulses and the stroke index at AT both in the control and Fontan groups. These results suggest that impaired exercise capacity in Fontan patients is mainly due to a subnormal response of the stroke index at AT and to the decreased response of the stroke index and the heart rate at the maximal workload. (Jpn Heart J 1998; 39: 659-669)

Key words: Fontan operation, Exercise, Anaerobic threshold, Oxygen consumption, Stroke index

$\mathrm{T}$ HE Fontan operation has been widely accepted as a surgical intervention for a variety of congenital cardiac malformations. Although exercise performance in patients after Fontan operation is improved when compared with conditions before repair, it is still reduced when compared with that of normal subjects. ${ }^{1-3)}$ Several reports have shown subnormal cardiac output during exercise testing in patients after Fontan operation. ${ }^{1,2.7)}$ Oxygen consumption as an index

From the Department of Cardiothoracic Surgery, Faculty of Medicine, Tokyo University, Tokyo, 'Department of Cardiothoracic Surgery, Funabashi Futawa Hospital, Chiba, and 'Department of Cardiothoracic Surgery, Japan Railway General Hospital, Tokyo, Japan.

Address for Correspondence: Takeshi Miyairi, MD, Department of Cardiothoracic Surgery, Faculty of Medicine, Tokyo University, 7-3-1 Bunkyo-ku, Tokyo 113-8655, Japan.

Received for publication July 6, 1998.

Revised and accepted August 10, 1998. 
of cardiorespiratory performance during exercise has also been studied, ${ }^{1,2,8-11)}$ but it has not been well addressed in relation to the hemodynamic response. It is therefore the purpose of this study to assess the hemodynamic changes and cardiorespiratory performance during upright bicycle exercise in patients after Fontan operation, and to clarify the relationship between them.

\section{Methods}

The study was performed on eight children who had undergone Fontan procedures. The preoperative anatomical diagnosis and previous procedures of the eight patients are summarized in Table I. Three patients were boys and five were girls. The mean age at operation was 6.1 years (range 2.6 to 8.3 ). The mean age at the time of study was 11.3 years (range 9.1 to 16.5 ) and the mean time since surgery was 5.2 years (range 2.8 to 8.5 ). In four patients an atriopulmonary anastomosis was performed. In two patients an atrioventricular connection had been created and in two patients total cavopulmonary anastomosis was constructed at surgery. Six patients were in New York Heart Association (NYHA) class I and two in class II.

The control group consisted of 10 patients who had undergone closure of the atrial septal defect more than 5 years before. The mean age of the control group was 13.4 years (range 10.3 to 18.8). Seven patients were boys and three were girls. All patients were in NYHA class I and were engaged in normal daily

Table I. Clinical Profile of the Patients after Fontan Operation

\begin{tabular}{|c|c|c|c|c|c|c|c|c|}
\hline $\begin{array}{l}\text { Patient } \\
\text { no. }\end{array}$ & Sex & BSA & Diagnosis & $\begin{array}{l}\text { Previous } \\
\text { palliation }\end{array}$ & $\begin{array}{l}\text { Modified } \\
\text { Fontan } \\
\text { operation }\end{array}$ & $\begin{array}{c}\text { Age at } \\
\text { operation } \\
\text { (yr) }\end{array}$ & $\begin{array}{c}\text { Age at } \\
\text { study } \\
(\mathrm{yr})\end{array}$ & $\begin{array}{c}\text { Time } \\
\text { from } \\
\text { operation } \\
\text { (yr) }\end{array}$ \\
\hline 1 & $M$ & 1.06 & TA & Waterston, ASDcre & Molina & 8.3 & 13.3 & 5 \\
\hline 2 & $\mathrm{~F}$ & 1.22 & SV & $\mathrm{PAB}$ & Molina & 5.2 & 10.4 & 5.2 \\
\hline 3 & $\mathrm{~F}$ & 1.06 & TA, ASD, VSD, PS & right-BT & Molina & 5.8 & 9.2 & 3.4 \\
\hline 4 & $\mathbf{M}$ & 1.14 & TA, PS & left-BT & Molina & 6.8 & 11.8 & 5 \\
\hline 5 & $\mathbf{F}$ & 1.03 & TA, PDA, WPW & & Bjork & 5.6 & 10.6 & 5 \\
\hline 6 & $\mathrm{~F}$ & 1.51 & TA, VSD, PS & left-BT & Bjork & 8 & 16.5 & 8.5 \\
\hline 7 & $M$ & 1.09 & $\begin{array}{l}\text { SA, SV, PDA, PA, d-TGA, } \\
\text { CAVVR }\end{array}$ & & TCPC & 2.6 & 9.5 & 6.9 \\
\hline 8 & F & 0.98 & $\begin{array}{l}\text { SA, SV, PDA, PS, d-TGA, } \\
\text { bil-SVC }\end{array}$ & left $\bmod B T$ & TCPC & 6.3 & 9.1 & 2.8 \\
\hline Mean & & 1.14 & & & & 5.9 & 11.1 & 5.3 \\
\hline
\end{tabular}

$\mathrm{M}=$ male; $\mathrm{F}=$ female; bil- = bilateral-; $\mathrm{TA}=$ tricuspid atresia; $\mathrm{SV}=$ single ventricle; $\mathrm{ASD}=$ atrial septum defect; VSD = ventricular septum defect; PS = pulmonary stenosis; PDA = patent ductus arteriosus; WPW $=$ Wolff-Parkinson-White syndrome; $\mathrm{SA}=$ single atrium; PA = pulmonary atresia; $\mathrm{TGA}=$ transposition of great arteries; CAVVR = common atrioventricular valve regurgitation; $\mathrm{ASD}$ cre $=\mathrm{ASD}$ creation; $\mathrm{PAB}=$ pulmonary artery banding; $\mathrm{BT}=$ Blalock-Taussig anastomosis; $\bmod =\operatorname{modified} ; \mathrm{TCPC}=$ total cavopulmonary connection. 
activities. Two dimensional and Doppler echocardiography at rest showed normal ejection fraction of the left ventricle $(>70 \%)$ without any residual shunts in these patients.

Study protocol: Before exercise testing, each patient had a thorough physical examination, including a resting 12-lead ECG, a chest roentgenogram and echocardiography (Sonolayer Model SSH-65A with $3.5-\mathrm{MHz}$ and $2.5-\mathrm{MHz}$ transducer, Toshiba Corp., Japan).

A $3.5-\mathrm{MHz}$ phased-array transducer was placed in the left parasternal position to record a long axis view of the left ventricle and aortic valve.The aortic diameter was measured at the annulus during systole by M-mode echocardiography. The cross-sectional area of the aorta was calculated from the aortic diameter on the assumption that the aorta is circular.

Before the exercise study, the procedure was carefully explained and informed consent was obtained in each case from the patients' parents or legal guardian.

The exercise testing was performed with a calibrated electronically-braked bicycle ergometer (Ergometer 232C, Combi, Tokyo). The patients underwent supine exercise with the workload beginning at 0 watts and increasing continuously in increments of 10 watts per minute. Heart rate, electrocardiogram and blood pressure were continuously monitored throughout the test. The exercise protocol was continued to the limit of tolerance. No evidence of ischemia, ventricular arrhythmia or systemic hypotension was found in any of the patients before they had reached tolerance.

The subjects exhaled through a low-resistance one-way valve and a pneumotachometer into a mixing chamber. Mixed expired gas was continuously sampled and analyzed for oxygen and carbon dioxide by an electronic gas analyzer (Mets-900, Vise Medical Ltd., Japan). Oxygen uptake, carbon dioxide production, minute ventilation, and the ventilatory equivalent for oxygen were calculated breath-by-breath by an on-line computer (PC-9801, NEC Ltd. Japan). ${ }^{12)}$ The anacrobic threshold (AT) was determined by gas exchange criteria as the point of a nonlinear increase in the minute ventilation for workload. This onset of hyperventilation was further confirmed by referring to the exercise intensity at which the carbon dioxide production increased nonlinearly for the oxygen uptake. This point was determined by one well-trained investigator. To delineate more detailed development of the cardiorespiratory response, variables at the half intensity of the anaerobic threshold (1/2 AT) were also evaluated. Continuous wave Doppler recordings of ascending aortic flow were obtained with a small, dedicated $2.5 \mathrm{MHz}$ pencil-type transducer from the suprasternal notch. Transaortic flow was identified by its audio signal, spectral display and simultaneous imaging. The spectral envelopes of five consecutive cardiac cycles with the 
highest peak velocity were recorded and analyzed.

Calculations: The oxygen pulse was calculated as the oxygen uptake divided by the heart rate. The velocity integral was measured by averaging the planimetered area under the spectral display. The stroke index was calculated as the product of the velocity integral and aortic cross-sectional area divided by the body surface area. The cardiac index was calculated as the product of heart rate and stroke index.

Statistical analysis: Numerical data are expressed as mean $\pm 1 \mathrm{SD}$. Variables in the control and Fontan groups were compared using unpaired $t$-tests, and variables at rest, at anaerobic threshold and at peak exercise were compared using paired $t$-tests, with a $5 \%$ level of significance. The relation between SVI at AT (or the peak work load) and other variables was evaluated by linear regression using the method of least squares to obtain the best fit.

\section{Results}

Exercise testing was achieved to tolerance without adverse problems and the anaerobic threshold could be determined in all patients. Table II summarizes and compares performance variables at rest and during exercise in both groups.

The average $( \pm \mathrm{SD})$ oxygen consumption at rest expressed as values normalized for body weight was $4.2 \pm 0.4 \mathrm{~m} l / \mathrm{kg}$ in the control group and $4.2 \pm 0.4$ $\mathrm{m} l / \mathrm{kg}$ in the Fontan group (NS). During exercise, both groups showed a significant $(p<0.01)$ rise in oxygen consumption throughout exercise. However, the rate of increase in oxygen consumption was different between the two groups and

Table II. Summary of Progressive Exercise Test

\begin{tabular}{|c|c|c|c|c|}
\hline Group & $\begin{array}{l}\text { Oxygen consumption } \\
\qquad(\mathrm{m} / / \mathrm{min} / \mathrm{kg})\end{array}$ & $\begin{array}{l}\text { Heart rates } \\
\text { (beats } / \text { min) }\end{array}$ & $\begin{array}{l}\text { Cardiac index } \\
\left(\mathrm{L} / \mathrm{min} / \mathrm{m}^{2}\right)\end{array}$ & $\begin{array}{c}\text { Stroke index } \\
\left(\mathrm{m} / / \mathrm{min} / \text { beat } / \mathrm{m}^{2}\right)\end{array}$ \\
\hline \multicolumn{5}{|l|}{ Fontan $(n=8)$} \\
\hline rest & $4.2 \pm .4$ & $83 \pm 9$ & $4.1 \pm .5$ & $49.2 \pm 4.2$ \\
\hline AT & $15.6 \pm 1.5^{*}$ & $132 \pm 12^{*}$ & $7.7 \pm .7 *$ & $58.7 \pm 2.0^{*}$ \\
\hline Max & $19.8 \pm 3.4^{* * * *}$ & $163 \pm 11^{* * * *}$ & $8.8 \pm .9^{*, * *}$ & $53.8 \pm 4.4^{* .}$ \\
\hline \multicolumn{5}{|c|}{ Control $(n=10)$} \\
\hline rest & $4.2 \pm .4$ & $77 \pm 5$ & $3.8 \pm .5$ & $49.4 \pm 4.2$ \\
\hline AT & $20.3 \pm 3.3^{*}$ & $128 \pm 7^{*}$ & $8.8 \pm .8^{*}$ & $69.1 \pm 7.7^{*}$ \\
\hline Max & $32.3 \pm 7.2^{* * * *}$ & $174 \pm 6^{*, * *}$ & $11.9 \pm 1.7^{*, * *}$ & $68.4 \pm 9.7^{*}$ \\
\hline \multicolumn{5}{|c|}{ Difference between groups } \\
\hline rest & NS & NS & NS & NS \\
\hline $\mathrm{AT}$ & $p<0.01$ & NS & $p<0.01$ & $p<0.01$ \\
\hline Max & $p<0.001$ & $p<0.05$ & $p<0.001$ & $p<0.01$ \\
\hline
\end{tabular}


oxygen consumption at each stage of exercise was significantly $(p<0.01)$ lower in the Fontan group compared with the control subjects.

The heart rates at rest were $77 \pm 5$ beats/min in the control group and $83 \pm 9$ beats/min in the Fontan group (NS). Up to the anaerobic threshold, the slopes of the heart rate response to exercise did not differ between the two groups. From AT to the peak work load, the increments in heart rates were different between the two groups and heart rates at the peak work load were significantly $(p<0.05)$ lower in the Fontan group compared with the control group.

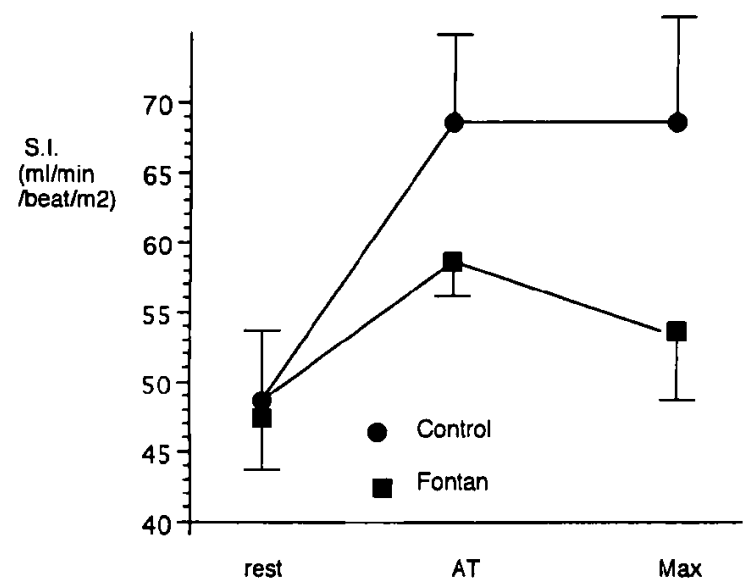

Figure 1. Changes in stroke index of the Fontan group and the control group at rest and during exercise.

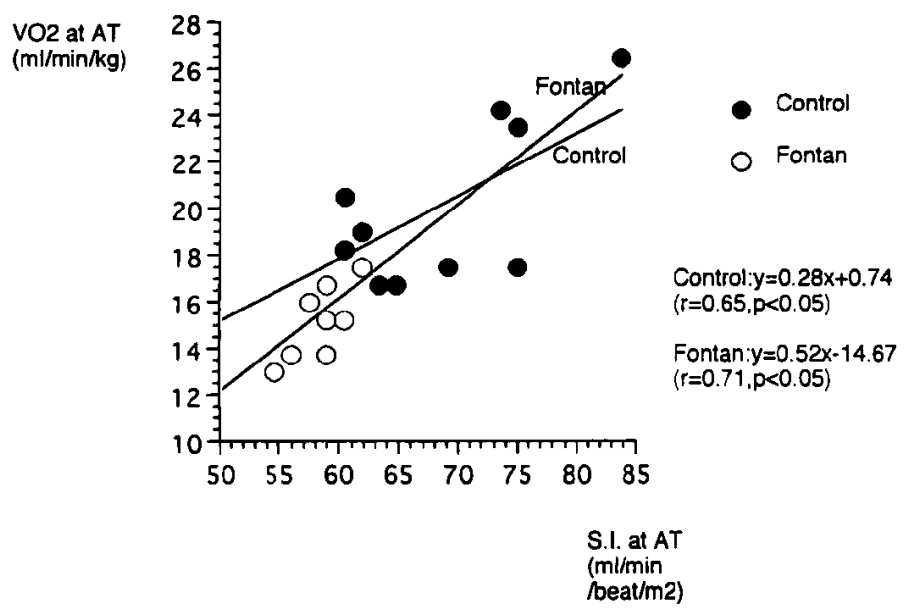

Figure 2. Relationship between stroke index and oxygen consumption at the anaerobic threshold of the Fontan and control groups. 


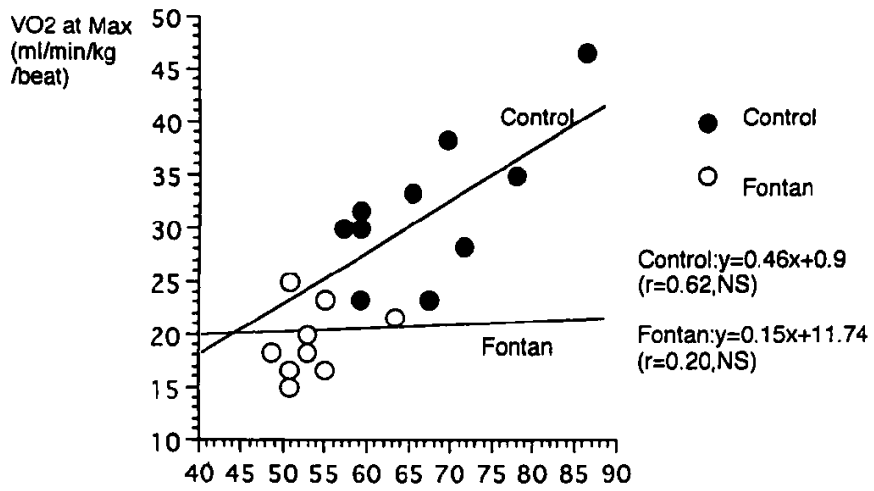

$$
\begin{aligned}
& \text { S.I. at Max } \\
& \text { (ml/min/beat } \\
& \text { /m2) }
\end{aligned}
$$

Figure 3. Relationship between stroke index and oxygen pulses at the anaerobic threshold of the Fontan and control groups.

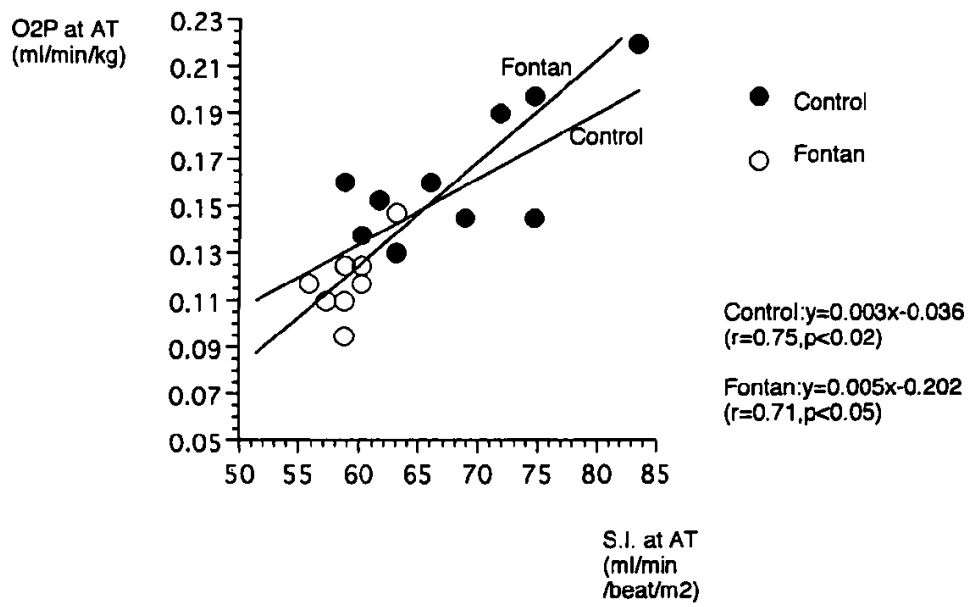

Figure 4. Relationship between stroke index and oxygen consumption at the peak workload of the Fontan and control groups.

The cardiac index at rest was similar for the control and Fontan groups, $3.8 \pm 0.5$ and $4.1 \pm 0.5 \mathrm{l} / \mathrm{min} / \mathrm{m}^{2}$, respectively. During the transition from rest to AT, cardiac index increased at a similar rate in both groups, increasing signifcantly $(p<0.01)$ at each exercise stage. From AT to the peak work load, although the increase in the cardiac index was significant $(p<0.01)$ in both groups, it was more modest in the Fontan group and the cardiac index reached at the peak workload was significantly $(p<0.001)$ lower in the Fontan group compared with the control group.

The stroke index at rest was comparable for the two groups, $49.4 \pm 4.2 \mathrm{ml}$ 


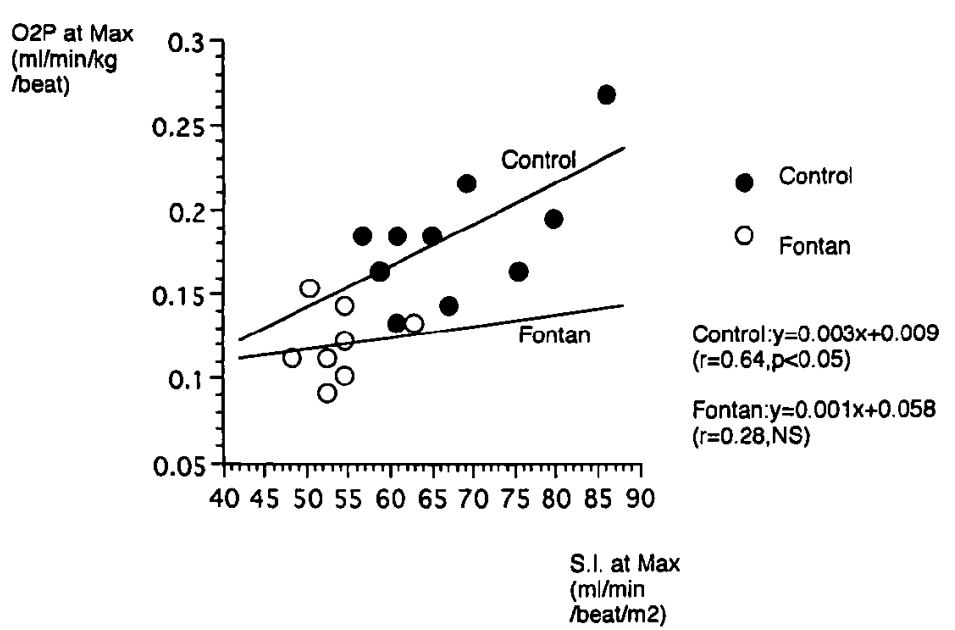

Figure 5. Relationship between stroke index and oxygen pulses at the peak workload of the Fontan and control groups.

$\mathrm{min} / \mathrm{m}^{2} /$ beats in the control group and $49.2 \pm 4.2 \mathrm{ml} / \mathrm{min} / \mathrm{m}^{2} /$ beats in the Fontan group (NS). At both 1/2 AT and AT, the stroke index was increased significantly $(p<0.01)$ compared with the resting values. At the peak work load, the stroke index remained almost at the same level as that of AT in the control group and had significantly decreased in the Fontan group $(p<0.05)$ (Figure 1). The mean value of this index at the peak workload was increased by $9.3 \%$ $(p<0.05)$ in the Fontan group and by $38.4 \%(p<0.01)$ in the control group compared with the resting values. The stroke index at the peak workload was lower than the resting value in 2 of 8 Fontan patients $(25 \%)$. In the control subjects, however, these phenomenon were not observed.

The oxygen consumption was significantly correlated with the stroke index at AT both in the Fontan group $(r=0.71, p<0.05)$ and in the control group $(r=0.65, p<0.5)$ (Figure 2). At the peak workload, no correlations were observed between oxygen consumption and stroke index for either group (Figure 3).

Significant correlations were observed between the oxygen pulses and the stroke index at AT both in the control group $(r=0.75, p<0.02)$ and the Fontan group $(r=0.71, p<0.05)$ (Figure 4). Correlation coefficients between the oxygen pulses and stroke index at the peak workload for the Fontan and control groups were $0.28(\mathrm{NS})$ and $0.64(p<0.05)$, respectively (Figure 5).

\section{Discussion}

Exercise testing has been widely used to evoke myocardial ischemia in 
patients with ischemic heart disease. It has also been performed to elicit an abnormality in ventricular function in patients with different cardiac conditions. Although maximal exercise testing has been reported to provide an objective assessment of functional capacity, it can be biased by the subject's motivation because the test is discontinued according to the symptoms of each subject. Moreover, exercise testing to maximal tolerance may include potential hazards in patients with severe heart disease.

The anaerobic threshold, which is the workload when the oxygen delivery to working muscles cannot increase enough to maintain aerobic metabolism during progressive exercise, has been proposed as an alternate means to quantify exercise capacity. ${ }^{13-16)}$ This onset of anaerobic metabolism has been found to be detected noninvasively by respiratory measurement of expired gas during incremental exercise testing, ${ }^{17)}$ though the method of determining the anaerobic threshold is still controversial.

The anaerobic threshold can be detected well before the maximal effort irrespective of the subject's motivation. The ratio of the anaerobic threshold to the maximal oxygen consumption has been described as $50-60 \%$ in normal subjects $^{14,15,18)}$ and approximately $70 \%$ in cardiac patients. ${ }^{19,20)}$ Previous reports have demonstrated that the anaerobic threshold was an accurate and reproducible index to classify the severity of an impairment of cardiac function. ${ }^{19-21)}$ Therefore, it is natural that this index of submaximal exercise has also been applied to patients with congenital cardiac defects. ${ }^{22-25}$

Several investigators have reported that patients after Fontan procedure had impaired exercise capacity. ${ }^{1,2,411)}$ Zellers et al ${ }^{1 /}$ described that oxygen consumption at the peak workload for patients after Fontan procedure was approximately $60 \%$ of predicted values. In the present study the mean oxygen consumption at the peak workload for the Fontan group was $61 \%$ of that of the control group. Yasui ${ }^{3}$ found oxygen consumption at the anaerobic threshold for Fontan patients to average approximately $80 \%$ of normal, similar to that documented in this study. The heart rate response to exercise was consistent with previous observations. The heart rate was comparable for both groups at each exercise stage until AT. However, the maximal heart rate reached at the peak workload was significantly lower in the Fontan group compared with the control group. ${ }^{2,3,5,79)}$

Previous studies have demonstrated that the cardiac output response to exercise was below normal in Fontan patients. ${ }^{1,2,4-6)}$ This subnormal response of cardiac output has been attributed to a notably subnormal stroke volume response and a slightly decreased heart rate response, and considered to be the main reason for the impaired exercise capacity of Fontan patients. Gewillig $e t a l^{b}$. reported that the cardiac index of Fontan patients maintained an ascending curve up to a submaximal effort point of $1.5 \mathrm{watt} / \mathrm{kg}$. Cortes $e t$ al $l^{7)}$ reported that 
the cardiac index of Fontan patients hardly changed from the 6th minute of the Bruce protocol incremental treadmill exercise because of the significant decrease in the stroke index and the lower response of the heart rate, while that of the control group continued to increase until the end of the maximal effort. In our study, the cardiac index of the Fontan group increased significantly at each stage till the point of maximal effort, though the increase after AT was substantially subnormal. The difference between the two groups is probably due to the maximal workload attained. With our continuous protocol of exercise testing, it is often uncertain whether the subject reached the oxygen plateau when they discontinued exercise due to symptoms. We concede that we may have underestimated the maximal workload in several patients. Cortes $e t a l^{7:}$ reported that the stroke index for Fontan patients decreased significantly at the point of maximal effort after reaching its peak value at the submaximal effort point. Our findings essentially agree with this observation. In our study, the stroke index for Fontan patients showed a significant increase (although subnormal) at each exercise stage until AT and significantly decreased at the peak workload.

The relationship between respiratory gas exchange and cardiac function during exercise is relatively unclear. This study is unique because the values of oxygen utilization and cardiac function were measured and compared at each stage in the same exercise testing.

At AT, significant correlations were observed between the oxygen pulses and the stroke index both in the control and Fontan groups. This means that the oxygen pulses well reflect and, thereby, can be a strong indicator of the stroke index at AT for both groups. Accordingly, oxygen consumption was considered to be a strong predictor of cardiac output at AT for both groups.

Since oxygen consumption can be expressed as the product of the cardiac output and the atriovenous oxygen $\left(\mathrm{AVO}_{2}\right)$ difference that was not directly measured in this study, the slope of the linear regression between the stroke index and oxygen pulses reflects the values of $\mathrm{AVO}_{2}$ difference. The values of the slope at AT were higher in the Fontan group than in the control group, which means the values of $\mathrm{AVO}_{2}$ difference at $\mathrm{AT}$ is greater in the Fontan group than in the control group, as earlier described by Shachar et al. ${ }^{4)}$ In addition, the heart rate was comparable for both groups at AT. Therefore, a subnormal response of the stroke index was considered to be the main reason for the decreased oxygen utilization at AT for Fontan patients.

At the peak workload, however, significant correlations between the oxygen pulses and the stroke index were observed only in the control group. The values of the slope of linear regression between stroke index and oxygen pulses at the peak workload were as high as those at AT. This means that the oxygen pulses can be an indicator of the stroke index also at the peak workload in the control 
subjects, and that the values of $\mathrm{AVO}_{2}$ difference at max are as high as those at $\mathrm{AT}$ in the control subjects, as earlier described by Rowell et al. ${ }^{26)}$ In contrast, the oxygen pulses can not be an indicator of the stroke index in the Fontan group possibly because of the diverse values of $\mathrm{AVO}_{2}$ difference at the peak workload in Fontan patients. Since the maximal heart rate is significantly lower in the Fontan patients than in the control subjects, the impaired oxygen utilization at the peak workload in Fontan patients is attributable to the subnormal increases in the stroke index and heart rate.

In conclusion, the present study has shown that the patients after Fontan procedure had impaired exercise capacity during upright ergometer exercise testing. At AT, the subnormal response of the stroke index was considered to be the main reason for the decreased exercise capacity in Fontan patients. In addition, the oxygen pulses at AT well reflected, and thereby, can be a strong indicator of the stroke index at AT in Fontan patients. Accordingly, oxygen consumption was considered to be a strong predictor of cardiac output at AT in Fontan patients. At maximal workload, the decreased exercise capacity for Fontan patients was commensurate with the impaired responses of the stroke index and heart rate.

\section{REFERENCES}

1. Zellers TM, Driscoll DJ, Mottream CD, Puga FJ, Schaff HV, Danielson GK. Exercise tolerance and cardiorespiratory response to exercise before and after the Fontan operation. Mayo Clin Proc 1989; 64: 1489-97.

2. Driscoll DJ, Danielson GK, Puga FJ, Schaff HV, Herse CT, Staats BA. Exercise tolerance and cardiorespiratory response to exercise after the Fontan operation for tricuspid atresia or functional single ventricle. J Am Coll Cardiol 1986; 7: 1087-94.

3. Yasui K. Cardiorespiratory response to exercise and anaerobic threshold before and after the Fontan procedure. Acta Cardiol Paed Jpn 1993;9: 295-302.

4. Shachar GB, Fuhrman BP, Wang Y, Lucas RV, Lock JE. Rest and exercise hemodyamics after the Fontan procedure. Circulation 1982;65: 1043-8.

5. Cumming GU. Maximal exercise hemodynamics after the Fontan procedure. In: Doyle EF, editor. Pediatric Cardiology. New York: Springer-Verlag, 1986; 257-66.

6. Gewillig $\mathrm{MH}$, Lundstrom UR, Bull C, Wyse RKH, Deanfield JE. Exercise responses in patients with congenital heart disease after Fontan repair: patterns and determinants of performance. $\mathrm{J}$ Am Coll Cardiol 1990;15: 1424-32.

7. Cortes RGS, Satomi G, Yoshigi M, Momma K. Maximal hemodynamic response after the Fontan procedure: Doppler evaluation during the treadmill test. Pediatr Cardiol 1994;15: 170-7.

8. Wessel HU, Paul MH. Impaired exercise performance in tricuspid atresia after Fontan operation or palliation by shunt (abstr.). Pediatr Cardiol 1983;4:76-7.

9. Driscoll DJ, Feldt RH, Mottram CD, Puga FJ, Schaff HV, Danielson GK. Cardiorespiratory response to exercise after definitive repair of univentricular atrioventricular connection. Int $\mathrm{J}$ Cardiol $1987 ; 17$ : 73-81.

10. Grant GP, Mansell AL, Garofano RP, Hayes CJ, Bowman FO, Gersony WM. Cardiorespiratory response to exercise afier the Fontan procedure for tricuspid atresia. Pediatr Res 1988;24: 1-5.

11. Rhodes J, Garofano RP, Bowman FO, Grant GP, Bierman FZ, Gersony WM. Effect of right ventricular anatomy on the cardiopulmonary response to exercise: implications for the Fontan procedure. Circulation 1990;81: 1811-7. 
12. Yamamoto Y. Takei Y, Mokushi K Breath-by-breath measurement of alveolar gas exchange with a slow response gas analyzer. Med Biol Eng Comput 1987; 25: 141.

13. Wasserman K, Mcllroy MB. Detecting the threshold of anaerobic metabolism in cardiac patients during exercise. Am J Cardiol 1964; 14: 844-52.

14. Wasserman K, Whipp BJ, Koyal SN, Beaver WL. Anaerobic threshold and respiratory gas exchange during exercise. J Appl Physiol 1973; 35:236-43.

15. Wasserman K, Whipp BJ. Exercise physiology in health and disease. Am Rev Respir Dis 1975; 112: 219-49.

16. Wasserman $\mathrm{K}$. The anaerobic threshold measurement in exercise testing. Clin Chest Med 1984; 5: 77-88.

17. Beaver W'L, Wasserman $\mathrm{K}$, Whipp BJ. A new method for detecting anaerobic threshold by gas exchange. J Appl Physiol 1986; 60:2020-7.

18. Powers SK, Dodd S, Garner R. Precision of ventilatory and gas exchange alterations as a predictor of the anaerobic threshold. Eur J Appl Physiol 1984;52: 173-7.

19. Weber KT, Kinasewitz GT, Janicki JS, Fishman AP. Oxygen utilization and ventilation during exercise in patients with chronic cardiac failure. Circulation 1982;675: 1213-23.

20. Matsumura N, Nishijima H, Kojima S, Hashimoto F, Minami M, Yasuda H. Determination of anaerobic threshold for assessment of functional state in patients with chronic heart failure. Circulation 1983; 68 : 360-7.

21. Kawauchi M, Matsunaga H, Miyairi T, Morizuki O, Furuse A. Oxygen uptake during exercise before and after cardiac valve surgery: anaerobic threshold and maximal oxygen uptake. Nippon-Kyobu-GekaGakkai-Zasshi (J Jpn Assc Thorac Surg) 1990; 38: 106-10 (in Japanese).

22. Reybrouck T, Weymans M, Stijns H, Van der Hauwaert LG. Exercise testing after correction of tetralogy of Fallot: the fallacy of a reduced heart rate response. Am Heart J 1986; 112: 998-1003.

23. Reybrouck T, Weymans M, Stijns H, Van der Hauwaert LG. Ventilatory anaerobic threshold for evaluating exercise performance in children with congenital left-to-right intracardiac shunt. Pediatr Cardiol 1986; 7: 19-24.

24. Reybrouck T, Dumoulin M, Van der Hauwaert LG. Cardiorespiratory exercise testing after venous switch operation in children with complete transposition of the great arteries. Am J Cardiol 1988; 61: 861-5.

25. Miyairi T, Kawauchi M, Furuse A. Exercise tolerance after correction of tetralogy of Fallot. KyobuGeka (Jpn J Thorac Surg) 1990; 43: 611-9 (in Japanese).

26. Rowell LB. Human cardiovascular control. New York: Oxford University Press, 1993; 162-203. 